\title{
GLOBAL SOLUTIONS FOR THE ONE DIMENSIONAL WATER-BAG MODEL*
}

\author{
MIHAI BOSTAN ${ }^{\dagger}$ AND JOSÉ ANTONIO CARRILLO $\ddagger$
}

\begin{abstract}
In this paper we study a special type of solution for the one dimensional VlasovMaxwell equations. We assume that initially the particle density is constant on its support in the phase space and we are looking for solutions where the particle density has the same property at any time $t>0$. More precisely, for each $x$ the support of the density is assumed to be an interval $\left[p^{-}, p^{+}\right]$ with end-points varying in space and time. Here we analyze the case of weak and strong solutions for the effective equations satisfied by the end-points and the electric field (water-bag model) in the relativistic setting.
\end{abstract}

Key words. Vlasov-Maxwell equations, water-bag model, conservation laws.

AMS subject classifications. 35A05, 78A35, 82D10.

\section{Introduction}

The Vlasov-Maxwell system governs the evolution of an ensemble of charged particles subject to electromagnetic fields created by themselves and possibly external sources in which collisions are typically neglected. Given $f$, the density number of charged particles at time $t \in \mathbb{R}_{+}$, position $x \in \mathbb{R}^{3}$ and momentum $p \in \mathbb{R}^{3}$, the dynamics of the particles is described by the Vlasov equation

$$
\partial_{t} f+v(p) \cdot \nabla_{x} f+q(E(t, x)+v(p) \wedge B(t, x)) \cdot \nabla_{p} f=0,(t, x, p) \in \mathbb{R}_{+} \times \mathbb{R}^{3} \times \mathbb{R}^{3},
$$

where the electromagnetic field $(E, B)$ is defined in a self-consistent way by the Maxwell equations

$$
\begin{gathered}
\partial_{t} E-c_{0}^{2} \operatorname{curl}_{x} B=-\frac{j(t, x)}{\varepsilon_{0}}, j(t, x)=q \int_{\mathbb{R}^{3}} v(p) f(t, x, p) d p,(t, x) \in \mathbb{R}_{+} \times \mathbb{R}^{3}, \\
\partial_{t} B+\operatorname{curl}_{x} E=0,(t, x) \in \mathbb{R}_{+} \times \mathbb{R}^{3}, \\
\operatorname{div}_{x} E=\frac{\rho(t, x)}{\varepsilon_{0}}, \rho(t, x)=q \int_{\mathbb{R}^{3}} f(t, x, p) d p, \operatorname{div}_{x} B=0,(t, x) \in \mathbb{R}_{+} \times \mathbb{R}^{3},
\end{gathered}
$$

where $q, m$ are the charge and the mass of the particles, $\varepsilon_{0}$ is the electric permittivity of the vacuum, and $v(p)$ is the relativistic velocity associated to the momentum $p$

$$
v(p)=\frac{p}{m}\left(1+\frac{|p|^{2}}{m^{2} c_{0}^{2}}\right)^{-\frac{1}{2}}
$$

where $c_{0}$ is the speed of light in a vacuum. Suitable initial conditions for the particle density and the electromagnetic field have to be prescribed satisfying certain compatibility conditions. The existence of global weak solutions was obtained in [10] and the existence of strong solutions has been investigated by different approaches in

${ }^{*}$ Received: April 30, 2008; accepted (in revised version): November 4, 2008. Communicated by François Bouchut.

†Laboratoire de Mathématiques de Besançon, UMR CNRS 6623, Université de Franche-Comté, 16 route de Gray, 25030 Besançon Cedex, France (mbostan@univ-fcomte.fr).

‡ICREA (Institució Catalana de Recerca i Estudis Avançats) and Departament de Matemàtiques, Universitat Autònoma de Barcelona, 08193 Bellaterra, Spain (carrillo@mat.uab.es). 
$[12,5,16]$. Despite these advances in existence theory for the Vlasov-Maxwell system, many questions concerning qualitative behavior, special solutions, and regularity issues, to name a few, are completely open. Recently global existence and uniqueness results have been proved for reduced models for laser-plasma interaction $[8,4]$, leading to particular global solutions of the Vlasov-Maxwell system.

Neglecting the magnetic field and the relativistic corrections in the Vlasov equation leads to the Vlasov-Poisson model

$$
\begin{array}{r}
\partial_{t} f+\frac{p}{m} \cdot \nabla_{x} f+q E(t, x) \cdot \nabla_{p} f=0,(t, x, p) \in \mathbb{R}_{+} \times \mathbb{R}^{3} \times \mathbb{R}^{3}, \\
\operatorname{curl}_{x} E=0, \operatorname{div}_{x} E=\frac{\rho(t, x)}{\varepsilon_{0}},(t, x) \in \mathbb{R}_{+} \times \mathbb{R}^{3},
\end{array}
$$

which is much better understood, see [1, 20, 17] for instance. The Vlasov-Poisson model can be justified as the limit of the relativistic Vlasov-Maxwell model when the characteristic speed of the particles remains small compared to the light speed $[9,3]$.

In this work, we elaborate on some particular type of solutions of the onedimensional version of the Vlasov-Maxwell(Poisson) system which has received the attention in the plasma physics community [2]. Let us assume that the initial density is proportional to the characteristic function of some region of the phase space between the graphs of two functions $p_{0}^{ \pm}: \mathbb{R} \rightarrow \mathbb{R}$

$$
f_{0}(x, p)=\alpha \mathbf{1}_{\left\{p_{0}^{-}(x)<p<p_{0}^{+}(x)\right\}},(x, p) \in \mathbb{R}^{2} .
$$

We assume that $p_{0}^{-} \leq p_{0}^{+}$. We are looking for a density function of the form

$$
f(t, x, p)=\alpha \mathbf{1}_{\left\{p^{-}(t, x)<p<p^{+}(t, x)\right\}},
$$

where $p^{ \pm}: \mathbb{R}_{+} \times \mathbb{R} \rightarrow \mathbb{R}$ are unknown functions to be determined such that the above density $f$ satisfies the Vlasov equation. We have the following immediate result:

Proposition 1.1. (Smooth water-bag solutions) Let $E:[0, T[\times \mathbb{R} \rightarrow \mathbb{R}$ be a given electric field which belongs to $L_{\text {loc }}^{1}\left(\left[0, T[\times \mathbb{R})\right.\right.$, with $0<T \leq+\infty$. Assume that $p^{ \pm}$: $\left[0, T\left[\times \mathbb{R} \rightarrow \mathbb{R}\right.\right.$ are smooth functions $p^{ \pm} \in W_{\text {loc }}^{1, \infty}([0, T[\times \mathbb{R})$ satisfying

$$
\left.\partial_{t} p^{ \pm}+v\left(p^{ \pm}\right) \partial_{x} p^{ \pm}=q E(t, x),(t, x) \in\right] 0, T[\times \mathbb{R},
$$

and $p^{-} \leq p^{+}$. Then the density $f$ given by (1.6) is a weak solution (that is, a solution in the sense of distributions) of the Vlasov equation associated with the electric field E.

Observe that the charge and current densities of the distribution in (1.6) are given by $\rho(t, x)=q \alpha\left(p^{+}(t, x)-p^{-}(t, x)\right), j(t, x)=q \alpha\left(\mathcal{E}\left(p^{+}(t, x)\right)-\mathcal{E}\left(p^{-}(t, x)\right)\right)$, where the kinetic energy function is given by

$$
\mathcal{E}(p)=m c_{0}^{2}\left(\left(1+\frac{p^{2}}{m^{2} c_{0}^{2}}\right)^{\frac{1}{2}}-1\right) .
$$

Note that we have $\mathcal{E}^{\prime}(p)=v(p)$. Thus for the initial condition in (1.5) the one dimensional Vlasov-Maxwell equations reduce to the system 


$$
\begin{gathered}
\left.\partial_{t} p^{ \pm}+\partial_{x} \mathcal{E}\left(p^{ \pm}\right)=q E(t, x),(t, x) \in\right] 0, T[\times \mathbb{R}, \\
\partial_{t} E=-\alpha \frac{q}{\varepsilon_{0}}\left(\mathcal{E}\left(p^{+}(t, x)\right)-\mathcal{E}\left(p^{-}(t, x)\right)\right), \\
\left.\partial_{x} E=\alpha \frac{q}{\varepsilon_{0}}\left(p^{+}(t, x)-p^{-}(t, x)\right),(t, x) \in\right] 0, T[\times \mathbb{R},
\end{gathered}
$$

with the initial conditions

$$
p^{ \pm}(0, x)=p_{0}^{ \pm}(x), E(0, x)=E_{0}(x), x \in \mathbb{R},
$$

satisfying

$$
E_{0}^{\prime}(x)=\alpha \frac{q}{\varepsilon_{0}}\left(p_{0}^{+}(x)-p_{0}^{-}(x)\right), p_{0}^{-}(x) \leq p_{0}^{+}(x), x \in \mathbb{R}
$$

Let us remark that (1.9) is a consequence of (1.7), (1.8) and the equality in (1.11). The problem (1.7), (1.8), (1.9), (1.10) is called the water-bag model and has been introduced in [2]. The idea is to reduce the Vlasov equation to a set of hydrodynamic equations while keeping its kinetic character. Aside from the transport of charged particles, such models arise in various domains. In [7] the inviscid Burgers equation is reduced to a closed system of moment equations, using a suitable concept of an entropy multivalued solution. Similar techniques for the reconstruction of a function from a finite number of moments apply in geometric optics computations $[14,15]$. The main goal of this paper is to establish existence and uniqueness results for the waterbag model. In section 2 we analyze the weak solutions: we study entropy solutions of the scalar conservation laws (1.7) coupled to equations (1.8), (1.9) for the electric field. Smooth solutions are constructed as well for certain class of initial conditions in section 3 .

\section{Weak solutions}

For simplicity we assume that all the physical constants $q, m, \varepsilon_{0}, c_{0}, \alpha$ are equal to unity. We remind the reader the standard existence and uniqueness results concerning the entropy solution for scalar conservation laws. We refer to $[13,11]$ for details on this topic. Here, we consider conservation laws with right hand side terms of the form

$$
\begin{gathered}
\partial_{t} u+\partial_{x} F(u)=G(t, x),(t, x) \in \mathbb{R}_{+} \times \mathbb{R}, \\
u(0, x)=u_{0}(x), x \in \mathbb{R} .
\end{gathered}
$$

THEOREM 2.1. (Entropy solutions for scalar conservation laws) Let us assume that $F: \mathbb{R} \rightarrow \mathbb{R}$ is a smooth function and $G$ belongs to $L_{\text {loc }}^{1}\left(\mathbb{R}_{+} ; L^{\infty}(\mathbb{R})\right)$. Then for any initial condition $u_{0} \in L^{\infty}(\mathbb{R})$ there is a unique entropy solution $u \in C\left(\mathbb{R}_{+} ; L_{\text {loc }}^{1}(\mathbb{R})\right) \cap$ $L_{\text {loc }}^{\infty}\left(\mathbb{R}_{+} ; L^{\infty}(\mathbb{R})\right)$ for $(2.1)$, (2.2) satisfying

$$
\|u(t)\|_{L^{\infty}(\mathbb{R})} \leq\left\|u_{0}\right\|_{L^{\infty}(\mathbb{R})}+\int_{0}^{t}\|G(s)\|_{L^{\infty}(\mathbb{R})} d s, t \in \mathbb{R}_{+} .
$$

Moreover if $v$ is the entropy solution associated to the initial condition $v_{0} \in L^{\infty}(\mathbb{R})$, the source term $H \in L_{\text {loc }}^{1}\left(\mathbb{R}_{+} ; L^{\infty}(\mathbb{R})\right)$, and the same smooth function $F$, then we have 
the inequality

$$
\begin{aligned}
\int_{\mathbb{R}}|u(t, x)-v(t, x)| \mathbf{1}_{\{|x|<R\}} d x \leq & \int_{\mathbb{R}}\left|u_{0}(x)-v_{0}(x)\right| \mathbf{1}_{\{|x|<R+t M(t)\}} d x \\
& +\int_{0}^{t} \int_{\mathbb{R}}|G(s, x)-H(s, x)| \mathbf{1}_{\{|x|<R+(t-s) M(t)\}} d x d s,
\end{aligned}
$$

where $M(t)=\max \left\{M_{u}(t), M_{v}(t)\right\}$,

$$
M_{u}(t)=\sup \left\{\left|F^{\prime}(\xi)\right|:|\xi| \leq\left\|u_{0}\right\|_{L^{\infty}(\mathbb{R})}+\int_{0}^{t}\|G(s)\|_{L^{\infty}(\mathbb{R})} d s\right\},
$$

and

$$
M_{v}(t)=\sup \left\{\left|F^{\prime}(\xi)\right|:|\xi| \leq\left\|v_{0}\right\|_{L^{\infty}(\mathbb{R})}+\int_{0}^{t}\|H(s)\|_{L^{\infty}(\mathbb{R})} d s\right\} .
$$

If $u_{0} \in B V(\mathbb{R})$ and $G \in L_{\text {loc }}^{1}\left(\mathbb{R}_{+} ; B V(\mathbb{R})\right)$ then the entropy solution has bounded variation and its total variation satisfies

$$
T V(u(t)) \leq T V\left(u_{0}\right)+\int_{0}^{t} T V(G(s)) d s, t \in \mathbb{R}_{+} .
$$

Furthermore, for any $t, R>0$ we have

$$
\begin{aligned}
\int_{\mathbb{R}}\left|u(t, x)-u_{0}(x)\right| \mathbf{1}_{\{|x|<R\}} d x \leq & t M_{u}(t)\left\{T V\left(u_{0}\right)+\int_{0}^{t} T V(G(s)) d s\right\} \\
& +2 R \int_{0}^{t}\|G(s)\|_{L^{\infty}(\mathbb{R})} d s
\end{aligned}
$$

It is well known that for conservation laws without a source term $(G=0)$, the solution operator $S(t) u_{0}=u(t, \cdot)$ is order preserving on $L^{1}(\mathbb{R}) \cap L^{\infty}(\mathbb{R})$ that is, for any $u_{0}, v_{0} \in$ $L^{1}(\mathbb{R}) \cap L^{\infty}(\mathbb{R})$ such that $u_{0} \leq v_{0}$ a.e. we have $S(t) u_{0} \leq S(t) v_{0}$ a.e. for any $t \in \mathbb{R}_{+}$. This is a direct consequence of the Crandall-Tartar lem. [13, page 81]. The same result holds for conservation laws with source terms $G \in L_{\text {loc }}^{1}\left(\mathbb{R}_{+} ; L^{\infty}(\mathbb{R})\right)$ and for initial conditions $u_{0} \in L^{\infty}(\mathbb{R})$.

LEMma 2.1. (Comparison principle with sources) Assume that the source $G \in$ $L_{\text {loc }}^{1}\left(\mathbb{R}_{+} ; L^{\infty}(\mathbb{R})\right)$ and denote by $S_{G}(t): L^{\infty}(\mathbb{R}) \rightarrow L^{\infty}(\mathbb{R})$ the solution operator given by $S_{G}(t) u_{0}=u(t, \cdot)$ for any $u_{0} \in L^{\infty}(\mathbb{R}), t \in \mathbb{R}_{+}$, where $u$ is the entropy solution of (2.1), (2.2). For any $t \in \mathbb{R}_{+}$the operator $S_{G}(t)$ is order preserving.

Proof. Since the solutions of (2.1) with bounded initial conditions propagate with finite speed (cf. $(2.3),(2.4)$ ), it is sufficient to prove the result for $G \in$ $L_{\text {loc }}^{1}\left(\mathbb{R}_{+} ; L^{1}(\mathbb{R})\right) \cap L_{\text {loc }}^{1}\left(\mathbb{R}_{+} ; L^{\infty}(\mathbb{R})\right)$ and initial conditions in $L^{\infty}(\mathbb{R}) \cap L^{1}(\mathbb{R})$. Therefore, consider $u_{0}, v_{0} \in L^{\infty}(\mathbb{R}) \cap L^{1}(\mathbb{R})$ such that $u_{0} \leq v_{0}$. We claim that

$$
\int_{\mathbb{R}}\left\{S_{G}(t) u_{0}-S_{G}(t) v_{0}\right\} d x=\int_{\mathbb{R}}\left\{u_{0}-v_{0}\right\} d x, t \in \mathbb{R}_{+} .
$$

Indeed, by (2.4) it is sufficient to prove it for compactly supported functions $u_{0}, v_{0}$ and this comes easily by interpreting $S_{G}(t) u_{0}, S_{G}(t) v_{0}$ as the limit of smooth solutions 
for approximating viscous problems, as the viscosity vanishes. We denote by $(\cdot)_{+}$the positive part function. Combining (2.4), (2.7) yields

$$
\begin{aligned}
2 \int_{\mathbb{R}}\left(S_{G}(t) u_{0}-S_{G}(t) v_{0}\right)_{+} d x & =\int_{\mathbb{R}}\left(S_{G}(t) u_{0}-S_{G}(t) v_{0}\right) d x+\int_{\mathbb{R}}\left|S_{G}(t) u_{0}-S_{G}(t) v_{0}\right| d x \\
& \leq \int_{\mathbb{R}}\left(u_{0}-v_{0}\right) d x+\int_{\mathbb{R}}\left|u_{0}-v_{0}\right| d x \\
& =2 \int_{\mathbb{R}}\left(u_{0}-v_{0}\right)_{+} d x=0,
\end{aligned}
$$

implying that $S_{G}(t) u_{0} \leq S_{G}(t) v_{0}$ a.e. $x \in \mathbb{R}, \forall t \in \mathbb{R}_{+}$.

Consider $p_{0}^{ \pm} \in L^{\infty}(\mathbb{R}), E_{0} \in L^{\infty}(\mathbb{R})$ satisfying $p_{0}^{-} \leq p_{0}^{+}$and $E_{0}^{\prime}=p_{0}^{+}-p_{0}^{-}$. We define the application $\mathcal{F}$ on $L_{\text {loc }}^{1}\left(\mathbb{R}_{+} ; L^{\infty}(\mathbb{R})\right)$ given by $\mathcal{F} E=E$ where

$$
\tilde{E}(t, x)=E_{0}(x)-\int_{0}^{t}\left\{\mathcal{E}\left(p^{+}(s, x)\right)-\mathcal{E}\left(p^{-}(s, x)\right)\right\} d s,
$$

and $p^{ \pm}$are the entropy solutions of

$$
\partial_{t} p^{ \pm}+\partial_{x} \mathcal{E}\left(p^{ \pm}\right)=E(t, x),(t, x) \in \mathbb{R}_{+} \times \mathbb{R},
$$

with the initial conditions $p_{0}^{ \pm}$. It is easily seen by (2.3) that

$$
\begin{aligned}
\|\tilde{E}(t)\|_{L^{\infty}(\mathbb{R})} & \leq\left\|E_{0}\right\|_{L^{\infty}(\mathbb{R})}+\int_{0}^{t}\left\{\left\|p^{+}(s)\right\|_{L^{\infty}(\mathbb{R})}+\left\|p^{-}(s)\right\|_{L^{\infty}(\mathbb{R})}\right\} d s \\
& \leq\left\|E_{0}\right\|_{L^{\infty}(\mathbb{R})}+t\left(\left\|p_{0}^{+}\right\|_{L^{\infty}(\mathbb{R})}+\left\|p_{0}^{-}\right\|_{L^{\infty}(\mathbb{R})}\right)+2 t \int_{0}^{t}\|E(s)\|_{L^{\infty}(\mathbb{R})} d s .
\end{aligned}
$$

For any $t \in \mathbb{R}_{+}$we denote by $e_{T}:[0, T] \rightarrow \mathbb{R}$ the function given by

$$
e_{T}(t)=\left(\left\|E_{0}\right\|_{L^{\infty}(\mathbb{R})}+T\left(\left\|p_{0}^{+}\right\|_{L^{\infty}(\mathbb{R})}+\left\|p_{0}^{-}\right\|_{L^{\infty}(\mathbb{R})}\right)\right) e^{2 T t} .
$$

We immediately check that the set $\mathcal{D}_{T}=\left\{E \in L^{1}(] 0, T\left[; L^{\infty}(\mathbb{R})\right):\|E(t)\|_{L^{\infty}(\mathbb{R})} \leq\right.$ $\left.e_{T}(t), \forall t \in[0, T]\right\}$ is left invariant by the application of $\mathcal{F}_{T}$ defined by $\mathcal{F}_{T} E=$ $\left.\mathcal{F} E\right|_{[0, T] \times \mathbb{R}}$ for any $E \in L_{\text {loc }}^{1}\left(\mathbb{R}_{+} ; L^{\infty}(\mathbb{R})\right)$.

A straightforward computation based on the contraction inequality (2.4) shows that $\mathcal{F}_{T}$ is continuous on $C\left([0, T] ; L_{\text {loc }}^{1}(\mathbb{R})\right)$. We denote by $M_{T}$ the constant given by

$$
M_{T}=\sup \left\{\left|\mathcal{E}^{\prime}(\xi)\right|:|\xi| \leq \max \left\{\left\|p_{0}^{-}\right\|_{L^{\infty}(\mathbb{R})},\left\|p_{0}^{+}\right\|_{L^{\infty}(\mathbb{R})}\right\}+\int_{0}^{T} e_{T}(t) d t\right\}<1 .
$$

Proposition 2.2. (Continuity of the map) Assume that $p_{0}^{ \pm}, E_{0} \in L^{\infty}(\mathbb{R})$. For any $T \in \mathbb{R}_{+}$we have the inequality

$$
\int_{\mathbb{R}}\left|\mathcal{F}_{T} E_{1}-\mathcal{F}_{T} E_{2}\right|(t, x) \mathbf{1}_{\{|x|<R\}} d x \leq 2 T \int_{0}^{t} \int_{\mathbb{R}}\left|E_{1}-E_{2}\right|(s, x) \mathbf{1}_{\left\{|x|<R+(t-s) M_{T}\right\}} d x d s
$$

for any $E_{1}, E_{2} \in \mathcal{D}_{T}, \forall t \in[0, T], R>0$.

Proof. Consider $E_{1}, E_{2} \in \mathcal{D}_{T}$ and let us denote the entropy solutions corresponding to the fields $E_{1}, E_{2}$ and the initial conditions $p_{0}^{ \pm}$by $p_{1}^{ \pm}, p_{2}^{ \pm}$. By the definitions of $\mathcal{F}_{T} E_{1}$, 
$\mathcal{F}_{T} E_{2}$ and (2.4) we easily deduce that

$$
\begin{aligned}
\int_{-R}^{R}\left|\left(\mathcal{F}_{T} E_{1}-\mathcal{F}_{T} E_{2}\right)\right|(t, x) d x & \leq \int_{0}^{t} \int_{-R}^{R}\left\{\left|p_{1}^{+}-p_{2}^{+}\right|(s, x)+\left|p_{1}^{-}-p_{2}^{-}\right|(s, x)\right\} d s \\
& \leq 2 \int_{0}^{t} \int_{0}^{s} \int_{\mathbb{R}}\left|E_{1}-E_{2}\right|(\tau, x) \mathbf{1}_{\left\{|x|<R+(s-\tau) M_{T}\right\}} d x d \tau d s \\
& \leq 2 T \int_{0}^{t} \int_{\mathbb{R}}\left|E_{1}-E_{2}\right|(s, x) \mathbf{1}_{\left\{|x|<R+(t-s) M_{T}\right\}} d x d s .
\end{aligned}
$$

Theorem 2.3. (Global entropy solutions for the water-bag model) Assume that $p_{0}^{ \pm}, E_{0} \in L^{\infty}(\mathbb{R})$ satisfying $E_{0}^{\prime}=p_{0}^{+}-p_{0}^{-}$. Then there is a unique global weak solution $\left(p^{+}, p^{-}, E\right) \in L^{\infty}(] 0, T[\times \mathbb{R})^{2} \times W^{1, \infty}(] 0, T[\times \mathbb{R}), \forall T \in \mathbb{R}_{+}$for the water-bag model (1.7), (1.8), (1.9), (1.10). Moreover if $p_{0}^{-} \leq p_{0}^{+}$then $p^{-} \leq p^{+}$.

Proof. It is sufficient to prove the existence of a unique solution $\left(p^{+}, p^{-}, E\right)$ on $[0, T] \times \mathbb{R}$ for any $T \in \mathbb{R}_{+}$. We define the sequence $\left(E^{n}\right)_{n \geq 0}$ given by $E^{0}(t, x)=E_{0}(x), \forall(t, x) \in[0, T] \times \mathbb{R}$ and $E^{n+1}=\mathcal{F}_{T} E^{n}, \forall n \in \mathbb{N}$. Observe that $\left(E^{n}\right)_{n} \subset$ $\mathcal{D}_{T}$. For any $R>0$ we consider the sequence of functions $z_{R}^{n}(t)=\int_{\mathbb{R}} \mid E^{n+1}-$ $E^{n} \mid(t, x) \mathbf{1}_{\left\{|x|<R+(T-t) M_{T}\right\}} d x, t \in[0, T], n \in \mathbb{N}$. By Proposition 2.2 it is easily seen that

$$
\begin{aligned}
z_{R}^{n}(t) & \leq 2 T \int_{0}^{t} \int_{\mathbb{R}}\left|E^{n}-E^{n-1}\right|(s, x) \mathbf{1}_{\left\{|x|<R+(T-s) M_{T}\right\}} d x d s \\
& =2 T \int_{0}^{t} z_{R}^{n-1}(s) d s, t \in[0, T], n \geq 1,
\end{aligned}
$$

implying that

$$
z_{R}^{n}(t) \leq \frac{(2 T t)^{n}}{n !}\left\|z_{R}^{0}\right\|_{L^{\infty}(] 0, T[)}, \forall n \in \mathbb{N} .
$$

We deduce that $\left(E^{n}\right)_{n}$ is a Cauchy sequence in $C\left([0, T] ; L_{\text {loc }}^{1}(\mathbb{R})\right)$ since

$$
\int_{\mathbb{R}}\left|E^{n+p}-E^{n}\right|(t, x) \mathbf{1}_{\{|x|<R\}} d x \leq z^{n}(t)+z^{n+1}(t)+\ldots+z^{n+p-1}(t) .
$$

It follows that $\left(E^{n}\right)_{n}$ converges in $C\left([0, T] ; L_{\text {loc }}^{1}(\mathbb{R})\right)$ towards a fixed point $E$ of $\mathcal{F}_{T}$. Moreover we easily check that $E \in \mathcal{D}_{T}$. Now take $p^{+}, p^{-}$to be the unique entropy solutions of (1.7) corresponding to the limit field $E$ and the initial conditions $p_{0}^{+}, p_{0}^{-}$. By construction $\left(p^{+}, p^{-}, E\right)$ is a solution for the water-bag model (1.7), (1.8), (1.10). Equation (1.9) is a consequence of (1.7), (1.8) and the constraint $E_{0}^{\prime}=p_{0}^{+}-p_{0}^{-}$. The bounds for the derivatives of $E$ come from the bounds of $p^{ \pm}$(see (2.3)) and (1.8), (1.9). For the inequality $p^{-} \leq p^{+}$, use Lemma 2.1. The uniqueness of the weak solution is obtained by a straightforward computation involving the Gronwall lemma.

Remark 2.1. (Vlasov-Maxwell solutions with defect measures) A natural question related to the previous existence result is the following: given $\left(p^{+}, p^{-}, E\right)$, a weak solution for the water-bag model, is it true that $f(t, x, p)=\mathbf{1}_{\left\{p^{-}(t, x)<p<p^{+}(t, x)\right\}}$ solves the Vlasov equation

$$
\partial_{t} f+v(p) \partial_{x} f+E(t, x) \partial_{p} f=0,(t, x, p) \in \mathbb{R}_{+} \times \mathbb{R} \times \mathbb{R} ?
$$


Generally the answer to this question is negative, but we can prove that $f$ solves a Vlasov equation with a entropy defect measure. Of course, here we appeal to the kinetic formulation of conservation laws $[18,19]$. Indeed, observe that the function $f$ can be represented as $f(t, x, p)=\chi\left(p, p^{+}(t, x)\right)-\chi\left(p, p^{-}(t, x)\right)$ where the function $\chi$ is given by

$$
\chi(\xi, u)=\left\{\begin{array}{c}
+1,0<\xi<u \\
-1, u<\xi<0 \\
0, \text { otherwise }
\end{array}\right.
$$

Since $p^{ \pm}$are entropy solutions we know that there the nonnegative kinetic entropy defect measures $m^{ \pm}$such that

$$
\left\{\begin{array}{l}
\partial_{t} \chi\left(p, p^{ \pm}\right)+v(p) \partial_{x} \chi\left(p, p^{ \pm}\right)-E(t, x) \delta_{0}\left(p-p^{ \pm}\right)=\partial_{p} m^{ \pm},(t, x, p) \in \mathbb{R}_{+} \times \mathbb{R}^{2}, \\
\chi\left(p, p^{ \pm}(0, x)\right)=\chi\left(p, p_{0}^{ \pm}(x)\right),(x, p) \in \mathbb{R}^{2},
\end{array}\right.
$$

where the notation $\delta_{0}$ stands for the Dirac mass concentrated at the origin. Therefore we obtain

$$
\left(\partial_{t}+v(p) \partial_{x}\right)\left\{\chi\left(p, p^{+}\right)-\chi\left(p, p^{-}\right)\right\}-E(t, x)\left\{\delta_{0}\left(p-p^{+}\right)-\delta_{0}\left(p-p^{-}\right)\right\}=\partial_{p}\left\{m^{+}-m^{-}\right\},
$$

and by taking into account that $\partial_{p}\left\{\chi\left(p, p^{+}\right)-\chi\left(p, p^{-}\right)\right\}=-\left\{\delta_{0}\left(p-p^{+}\right)-\delta_{0}\left(p-p^{-}\right)\right\}$ we can finally write

$$
\left\{\begin{array}{l}
\partial_{t} f+v(p) \partial_{x} f+E(t, x) \partial_{p} f=\partial_{p}\left\{m^{+}-m^{-}\right\},(t, x, p) \in \mathbb{R}_{+} \times \mathbb{R}^{2}, \\
f(0, x, p)=\chi\left(p, p_{0}^{+}(x)\right)-\chi\left(p, p_{0}^{-}(x)\right)=\mathbf{1}_{\left\{p_{0}^{-}(x)<p<p_{0}^{+}(x)\right\}},(x, p) \in \mathbb{R}^{2} .
\end{array}\right.
$$

Remark 2.2. (Total energy balance) Another interesting question concerns the behavior of the total energy given by

$$
W(t)=\int_{\mathbb{R}^{2}} \mathcal{E}(p) f d x d p+\frac{1}{2} \int_{\mathbb{R}} E(t, x)^{2} d x, t \in \mathbb{R}_{+} .
$$

For example if $p^{-}(t, x) \leq 0 \leq p^{+}(t, x),(t, x) \in[0, T] \times \mathbb{R}$, and the initial energy is finite, we can prove that the total energy is not increasing on $[0, T]$. Multiplying the above Vlasov equation by $\mathcal{E}(p)$ one obtains after integration

$$
\begin{aligned}
\frac{d}{d t} \int_{\mathbb{R}^{2}} \mathcal{E}(p) f d x d p-\int_{\mathbb{R}} E\left\{\mathcal{E}\left(p^{+}(t, x)\right)-\mathcal{E}\left(p^{-}(t, x)\right)\right\} d x= & \int_{\mathbb{R}^{2}} v(p) m^{-}(t, x, p) d x d p \\
& -\int_{\mathbb{R}^{2}} v(p) m^{+}(t, x, p) d x d p .
\end{aligned}
$$

Using (1.8) we also deduce that

$$
\frac{1}{2} \frac{d}{d t} \int_{\mathbb{R}} E(t, x)^{2} d x+\int_{\mathbb{R}} E(t, x)\left\{\mathcal{E}\left(p^{+}(t, x)\right)-\mathcal{E}\left(p^{-}(t, x)\right)\right\} d x=0,
$$

implying that

$$
\begin{aligned}
\frac{d}{d t}\left\{\int_{\mathbb{R}^{2}} \mathcal{E}(p) f d x d p+\frac{1}{2} \int_{\mathbb{R}} E(t, x)^{2} d x\right\}= & \int_{\mathbb{R}^{2}} v(p) m^{-}(t, x, p) d x d p \\
& -\int_{\mathbb{R}^{2}} v(p) m^{+}(t, x, p) d x d p .
\end{aligned}
$$


Therefore we are finished if we check that $m^{ \pm}=0$ on $[0, T] \times \mathbb{R} \times \mathbb{R}^{\mp}$. Take $p_{0}<0$ and let us multiply the kinetic formulation of $\chi\left(p, p^{+}\right)$by the derivative of the convex function $S_{p_{0}}(p)=\left(p-p_{0}\right)_{-}$. After standard computations involving the usual formula $\int_{\mathbb{R}} \chi(\xi, u) S^{\prime}(\xi) d \xi=S(u)-S(0), \forall S(\cdot), \forall u \in \mathbb{R}$, we obtain

$$
\frac{d}{d t} \int_{\mathbb{R}} S_{p_{0}}\left(p^{+}(t, x)\right) d x=-\int_{\mathbb{R}} m^{+}\left(t, x, p_{0}\right) d x .
$$

Therefore one obtains for any $t \in[0, T]$,

$$
\int_{\mathbb{R}} S_{p_{0}}\left(p^{+}(T, x)\right) d x+\int_{0}^{T} \int_{\mathbb{R}} m^{+}\left(t, x, p_{0}\right) d x d t=\int_{\mathbb{R}} S_{p_{0}}\left(p_{0}^{+}(x)\right) d x=0,
$$

implying that $m^{+}=0$ on $[0, T] \times \mathbb{R} \times \mathbb{R}^{-}$. In a similar way we check that $m^{-}=0$ on $[0, T] \times \mathbb{R} \times \mathbb{R}^{+}$.

REMARK 2.3. (Non-relativistic setting) This case is a little bit more difficult since the non relativistic energy function $\mathcal{E}(p)=\frac{p^{2}}{2}$ is only locally Lipschitz. This time the analog of estimate (2.8) becomes quadratic:

$$
\begin{aligned}
\|\tilde{E}(t)\|_{L^{\infty}} & \leq\left\|E_{0}\right\|_{L^{\infty}}+\frac{1}{2} \int_{0}^{t} \max \left\{\left\|p^{+}(s)\right\|_{L^{\infty}}^{2},\left\|p^{-}(s)\right\|_{L^{\infty}}^{2}\right\} d s \\
& \leq\left\|E_{0}\right\|_{L^{\infty}}+\int_{0}^{t}\left\{\max \left\{\left\|p_{0}^{+}\right\|_{L^{\infty}}^{2},\left\|p_{0}^{-}\right\|_{L^{\infty}}^{2}\right\}+s \int_{0}^{s}\|E(\tau)\|_{L^{\infty}}^{2} d \tau\right\} d s \\
& \leq\left\|E_{0}\right\|_{L^{\infty}}+t \max \left\{\left\|p_{0}^{+}\right\|_{L^{\infty}}^{2},\left\|p_{0}^{-}\right\|_{L^{\infty}}^{2}\right\}+t^{2} \int_{0}^{t}\|E(s)\|_{L^{\infty}}^{2} d s .
\end{aligned}
$$

In this case for $T>0$ small enough we denote by $e_{T}(\cdot)$ the unique solution of

$$
\frac{d}{d t} e_{T}=T^{2}\left(e_{T}(t)\right)^{2}, 0<t<T,
$$

with the initial condition $e_{T}(0)=\left\|E_{0}\right\|_{L^{\infty}}+T \max \left\{\left\|p_{0}^{+}\right\|_{L^{\infty}}^{2},\left\|p_{0}^{-}\right\|_{L^{\infty}}^{2}\right\}$. It is easily seen that the set $\mathcal{D}_{T}=\left\{E \in L^{1}(] 0, T\left[; L^{\infty}(\mathbb{R})\right):\|E(t)\|_{L^{\infty}} \leq e_{T}(t), \forall t \in[0, T]\right\}$ is left invariant by the application $\mathcal{F}_{T}$ and by following the same arguments as in the relativistic setting we construct a local unique weak solution $\left(p^{+}, p^{-}, E\right) \in L^{\infty}(] 0, T[\times \mathbb{R})^{2} \times$ $W^{1, \infty}(] 0, T[\times \mathbb{R})$ for the non relativistic water-bag model. For results on the multiwater-bag model in this setting see [6].

\section{Strong solutions}

This section is devoted to the analysis of smooth solutions for the relativistic water-bag model. We show that smooth nondecreasing initial conditions generate global smooth solutions.

Proposition 3.1. (Non-decreasing initial data for scalar conservation laws) Assume that $F \in W^{2, \infty}(\mathbb{R}), G \in L_{\text {loc }}^{\infty}\left(\mathbb{R}_{+} ; W^{1, \infty}(\mathbb{R})\right)$ such that $F^{\prime \prime} \geq 0, \partial_{x} G \geq 0$. Then for any nondecreasing initial condition $u_{0} \in W^{1, \infty}(\mathbb{R})$, problem $(2.1),(2.2)$ has a unique strong solution $u \in W^{1, \infty}(] 0, T[\times \mathbb{R}), \forall T \in \mathbb{R}_{+}$, which is nondecreasing with respect to $x$.

Proof. We define the sequence of functions $\left(u^{n}\right)_{n \geq 0}$ where $u^{0}(t, x)=u_{0}(x) \forall(t, x) \in$ $\mathbb{R}_{+} \times \mathbb{R}$ and for any $n \in \mathbb{N}, u^{n+1}$ solves the problem 


$$
\begin{gathered}
\partial_{t} u^{n+1}+F^{\prime}\left(u^{n}(t, x)\right) \partial_{x} u^{n+1}=G(t, x),(t, x) \in \mathbb{R}_{+} \times \mathbb{R}, \\
u^{n+1}(0, x)=u_{0}(x), x \in \mathbb{R} .
\end{gathered}
$$

In fact we will prove that $\left(u^{n}\right)_{n}$ are smooth and therefore the above problem is understood in the classical sense. Assume that $u^{n}$ belongs to $L_{\mathrm{loc}}^{\infty}\left(\mathbb{R}_{+} ; W^{1, \infty}(\mathbb{R})\right), \partial_{x} u^{n} \geq 0$, which is true for $n=0$, and let us show that the same holds for $u^{n+1}$. We denote by $X^{n}(s ; t, x)$ the characteristics associated to $F^{\prime}\left(u^{n}\right)$

$$
\frac{d}{d s} X^{n}(s ; t, x)=F^{\prime}\left(u^{n}\left(s, X^{n}(s ; t, x)\right)\right), X^{n}(t ; t, x)=x .
$$

Therefore we have

$$
u^{n+1}(t, x)=u_{0}\left(X^{n}(0 ; t, x)\right)+\int_{0}^{t} G\left(s, X^{n}(s ; t, x)\right) d s,(t, x) \in \mathbb{R}_{+} \times \mathbb{R} .
$$

We easily check that $u^{n+1} \in L_{\mathrm{loc}}^{\infty}\left(\mathbb{R}_{+} ; W^{1, \infty}(\mathbb{R})\right)$ and since $x \rightarrow X^{n}(s ; t, x), u_{0}$ and $G(s, \cdot)$ are nondecreasing and we deduce that $\partial_{x} u^{n+1} \geq 0$. Moreover we can find bounds for the time and space derivatives uniformly with respect to $n$. For any $h>0$ we have

$$
\begin{aligned}
& \partial_{t}\left\{u^{n+1}(t, x+h)-u^{n+1}(t, x)\right\}+\left\{F^{\prime}\left(u^{n}(t, x+h)\right)-F^{\prime}\left(u^{n}(t, x)\right)\right\} \partial_{x} u^{n+1}(t, x+h) \\
& +F^{\prime}\left(u^{n}(t, x)\right) \partial_{x}\left\{u^{n+1}(t, x+h)-u^{n+1}(t, x)\right\} \\
= & G(t, x+h)-G(t, x) .
\end{aligned}
$$

Since $\partial_{x} u^{n} \geq 0, \partial_{x} u^{n+1} \geq 0$, and $F^{\prime \prime} \geq 0$, we have

$$
\left\{F^{\prime}\left(u^{n}(t, x+h)\right)-F^{\prime}\left(u^{n}(t, x)\right)\right\} \partial_{x} u^{n+1}(t, x+h) \geq 0,
$$

and therefore

$$
\partial_{t} D_{h} u^{n+1}+F^{\prime}\left(u^{n}(t, x)\right) \partial_{x} D_{h} u^{n+1} \leq D_{h} G(t, x),
$$

where the notation $D_{h} z(x)$ stands for $z(x+h)-z(x)$ for any function $z$. Integrating along the characteristics one obtains

$$
D_{h} u^{n+1}(t, x) \leq\left(D_{h} u_{0}\right)\left(X^{n}(0 ; t, x)\right)+\int_{0}^{t} D_{h} G\left(s, X^{n}(s ; t, x)\right) d s,
$$

implying that

$$
\frac{D_{h} u^{n+1}(t, x)}{h} \leq\left\|u_{0}^{\prime}\right\|_{L^{\infty}(\mathbb{R})}+\int_{0}^{t}\left\|\partial_{x} G(s)\right\|_{L^{\infty}(\mathbb{R})} d s .
$$

Since we know that $\partial_{x} u^{n+1} \geq 0$ we finally obtain

$$
\left\|\partial_{x} u^{n+1}(t)\right\|_{L^{\infty}(\mathbb{R})} \leq\left\|u_{0}^{\prime}\right\|_{L^{\infty}(\mathbb{R})}+\int_{0}^{t}\left\|\partial_{x} G(s)\right\|_{L^{\infty}(\mathbb{R})} d s
$$

and

$$
\left\|\partial_{t} u^{n+1}(t)\right\|_{L^{\infty}(\mathbb{R})} \leq\|G(t)\|_{L^{\infty}(\mathbb{R})}+\left\|F^{\prime}\right\|_{L^{\infty}(\mathbb{R})}\left(\left\|u_{0}^{\prime}\right\|_{L^{\infty}(\mathbb{R})}+\int_{0}^{t}\left\|\partial_{x} G(s)\right\|_{L^{\infty}(\mathbb{R})} d s\right) .
$$


We claim that the sequence $\left(u^{n}\right)_{n}$ converges in $C([0, T] \times \mathbb{R}), \forall T \in \mathbb{R}_{+}$. Indeed, since $\left(\partial_{x} u^{n}\right)_{n}$ is bounded in $L^{\infty}(] 0, T[\times \mathbb{R})$, there is a constant $C_{T}$ depending on $\left\|u_{0}^{\prime}\right\|_{L^{\infty}(\mathbb{R})}$, $\int_{0}^{T}\left\|\partial_{x} G(s)\right\|_{L^{\infty}(\mathbb{R})} d s$, and $\left\|F^{\prime \prime}\right\|_{L^{\infty}}$ such that

$$
\left|X^{n+1}(s ; t, x)-X^{n}(s ; t, x)\right| \leq C_{T} \int_{s}^{t}\left\|u^{n+1}(\tau)-u^{n}(\tau)\right\|_{L^{\infty}} d \tau
$$

for any $(s, t, x) \in[0, T]^{2} \times \mathbb{R}$. Combining (3.3), (3.4) yields

$$
\left\|u^{n+2}(t)-u^{n+1}(t)\right\|_{L^{\infty}(\mathbb{R})} \leq \tilde{C}_{T} \int_{0}^{t}\left\|u^{n+1}(s)-u^{n}(s)\right\|_{L^{\infty}(\mathbb{R})} d s, n \in \mathbb{N},
$$

implying that the sequence $\left(u^{n}\right)_{n}$ converges in $C([0, T] \times \mathbb{R})$ towards some function $u$. Since $\left(\partial_{t} u^{n}\right)_{n},\left(\partial_{x} u^{n}\right)_{n}$ are bounded we deduce that $u \in W^{1, \infty}(] 0, T[\times \mathbb{R})$. It remains to prove that $u$ solves $(3.1),(3.2)$. There is a subsequence $\left(n_{k}\right)_{k}, \lim _{k \rightarrow+\infty} n_{k}=+\infty$ such that

$$
\lim _{k \rightarrow+\infty}\left(\partial_{t} u^{n_{k}}, \partial_{x} u^{n_{k}}\right)=\left(\partial_{t} u, \partial_{x} u\right), \text { weakly } \star \text { in } L^{\infty}(] 0, T[\times \mathbb{R})^{2} .
$$

Obviously we also have the convergence $\lim _{k \rightarrow+\infty} u^{n_{k}-1}=u$ in $C([0, T] \times \mathbb{R})$. Multiplying $(3.1)$ by a test function $\varphi \in C_{c}^{0}([0, T] \times \mathbb{R})$ one obtains

$$
\int_{0}^{T} \int_{\mathbb{R}} \partial_{t} u^{n_{k}} \varphi d x d t+\int_{0}^{T} \int_{\mathbb{R}} F^{\prime}\left(u^{n_{k}-1}(t, x)\right) \partial_{x} u^{n_{k}} \varphi d x d t=\int_{0}^{T} \int_{\mathbb{R}} G(t, x) \varphi(t, x) d x d t .
$$

We can easily pass to the limit for $k \rightarrow+\infty$ and obtain

$$
\int_{0}^{T} \int_{\mathbb{R}} \partial_{t} u \varphi d x d t+\int_{0}^{T} \int_{\mathbb{R}} F^{\prime}(u(t, x)) \partial_{x} u \varphi d x d t=\int_{0}^{T} \int_{\mathbb{R}} G(t, x) \varphi(t, x) d x d t
$$

showing that $u$ is a strong solution of (3.1). Moreover $u$ verifies the initial condition (3.2), since

$$
u(0, x)=\lim _{n \rightarrow+\infty} u^{n}(0, x)=u_{0}(x), x \in \mathbb{R} .
$$

Since any strong solution coincides with the entropy solution, we have also the uniqueness of the strong solution.

THEOREM 3.2. (Global smooth solutions) Assume that $p_{0}^{ \pm}, E_{0} \in W^{1, \infty}(\mathbb{R})$ satisfy $\frac{d}{d x} p_{0}^{ \pm} \geq 0, \frac{d}{d x} E_{0}=p_{0}^{+}-p_{0}^{-} \geq 0$. Then there is a global unique strong solution $\left(p^{+}, p^{-}, E\right) \in W^{1, \infty}(] 0, T[\times \mathbb{R})^{2} \times W^{2, \infty}(] 0, T[\times \mathbb{R}), \forall T \in \mathbb{R}_{+}$for the water-bag model.

Proof. By Theorem 2.3 we know that there is a global weak solution $\left(p^{+}, p^{-}, E\right) \in$ $L^{\infty}(] 0, T[\times \mathbb{R})^{2} \times W^{1, \infty}(] 0, T[\times \mathbb{R}), \forall T \in \mathbb{R}_{+}$, for the water-bag model satisfying $p^{-} \leq$ $p^{+}$. By (1.9) we have $\partial_{x} E \geq 0$ and by the definition the energy function $\mathcal{E}$ is convex. Thus applying Proposition 3.1 implies that the entropy solutions $p^{ \pm}$belong to $W^{1, \infty}(] 0, T[\times \mathbb{R})$ and are strong solutions for $(1.7)$. The bounds for the second order derivatives of the electric fields follow immediately from the bounds of the first order derivatives for $p^{ \pm}$and (1.8), (1.9). The uniqueness of the strong solution $\left(p^{+}, p^{-}, E\right)$ for the water-bag model is a direct consequence of the uniqueness of the weak solution. 
The previous theorem states the existence of smooth solutions $\left(p^{ \pm}, E\right)$ for the water-bag model, when the initial conditions $\left(p_{0}^{ \pm}, E_{0}\right)$ are nondecreasing Lipschitz continuous functions. In this case we know by Proposition 1.1 that the density $f(t, x, p)=\mathbf{1}_{\left\{p^{-}(t, x)<p<p^{+}(t, x)\right\}}$ is a weak solution of the Vlasov equation and therefore $(f, E)$ solves the Vlasov-Maxwell problem corresponding to the initial conditions $f_{0}(x, p)=\mathbf{1}_{\left\{p_{0}^{-}(x)<p<p_{0}^{+}(x)\right\}}$ and $E_{0}$. An interesting question is what happens when the initial conditions $p_{0}^{ \pm}$are not smooth. Is it true that the solutions of the VlasovMaxwell system and water-bag model satisfy $f(t, x, p)=\mathbf{1}_{\left\{p^{-}(t, x)<p<p^{+}(t, x)\right\}}$ ? The next theorem gives a partial affirmative answer to this question at least for nondecreasing initial conditions.

TheOREm 3.3. (Weak water-bag solutions) Assume that $p_{0}^{ \pm}, E_{0} \in L^{\infty}(\mathbb{R})$ are nondecreasing and satisfy $E_{0}^{\prime}=p_{0}^{+}-p_{0}^{-} \in L^{\infty}(\mathbb{R})$. We denote by $f_{0}: \mathbb{R}^{2} \rightarrow \mathbb{R}$ the function $f_{0}(x, p)=\mathbf{1}_{\left\{p_{0}^{-}(x)<p<p_{0}^{+}(x)\right\}}, \quad(x, p) \in \mathbb{R}^{2}$. Then there is a global unique weak solution $(f, E) \in L^{\infty}(] 0, T\left[\times \mathbb{R}_{x} ; L^{1}\left(\mathbb{R}_{p}\right)\right) \times W^{1, \infty}(] 0, T[\times \mathbb{R}), \forall T \in \mathbb{R}_{+}$for the VlasovMaxwell system corresponding to the initial conditions $\left(f_{0}, E_{0}\right)$. Moreover we have $f(t, x, p)=\mathbf{1}_{\left\{p^{-}(t, x)<p<p^{+}(t, x)\right\}}$ where $p^{ \pm}$are weak solutions for the water-bag model (1.7), (1.8), (1.9), (1.10).

Proof. We have $f_{0} \in L^{\infty}\left(\mathbb{R}_{x} ; L^{1}\left(\mathbb{R}_{p}\right)\right)$ and $E_{0} \in W^{1, \infty}(\mathbb{R})$ and thus there is a unique weak solution $(f, E) \in L^{\infty}(] 0, T\left[\times \mathbb{R}_{x} ; L^{1}\left(\mathbb{R}_{p}\right)\right) \times W^{1, \infty}(] 0, T[\times \mathbb{R}), \forall T \in \mathbb{R}_{+}$for the Vlasov-Maxwell system. The difficulty lies in checking that the density $f$ remains of the form $\mathbf{1}_{\left\{p^{-}(t, x)<p<p^{+}(t, x)\right\}}$, where $p^{ \pm}$weakly solve the conservation laws (1.7). Since this is true for smooth initial conditions, let us proceed by regularization. For any $\varepsilon>0$ consider $p_{0, \varepsilon}^{+}, p_{0, \varepsilon}^{-}, E_{0, \varepsilon} \in W^{1, \infty}(\mathbb{R})$, which are nondecreasing and satisfy

$$
\begin{gathered}
E_{0, \varepsilon}^{\prime}=p_{0, \varepsilon}^{+}-p_{0, \varepsilon}^{-},\left\|p_{0, \varepsilon}^{+}\right\|_{L^{\infty}} \leq\left\|p_{0}^{+}\right\|_{L^{\infty}},\left\|p_{0, \varepsilon}^{-}\right\|_{L^{\infty}} \leq\left\|p_{0}^{-}\right\|_{L^{\infty}},\left\|E_{0, \varepsilon}\right\|_{W^{1, \infty}} \leq\left\|E_{0}\right\|_{W^{1, \infty}} . \\
\lim _{\varepsilon \searrow 0}\left(p_{0, \varepsilon}^{+}, p_{0, \varepsilon}^{-}\right)=\left(p_{0}^{+}, p_{0}^{-}\right) \text {in } L_{\mathrm{loc}}^{1}(\mathbb{R})^{2}, \lim _{\varepsilon \searrow 0} E_{0, \varepsilon}=E_{0} \text { in } C^{0}(\mathbb{R}) .
\end{gathered}
$$

As before we know that there is a global unique weak solution for the VlasovMaxwell system $\left(f_{\varepsilon}, E_{\varepsilon}\right) \in L^{\infty}(] 0, T\left[\times \mathbb{R}_{x} ; L^{1}\left(\mathbb{R}_{p}\right)\right) \times W^{1, \infty}(] 0, T[\times \mathbb{R}), \forall T \in \mathbb{R}_{+}$associated to the initial conditions $f_{0, \varepsilon}(x, p)=\mathbf{1}_{\left\{p_{0, \varepsilon}^{-}(x)<p<p_{0, \varepsilon}^{+}(x)\right\}}$ and $E_{0, \varepsilon}$. By Theorem 3.2 a global unique strong solution $\left(p_{\varepsilon}^{+}, p_{\varepsilon}^{-}, \tilde{E}_{\varepsilon}\right) \in W^{1, \infty}(] 0, T[\times \mathbb{R})^{2} \times W^{2, \infty}(] 0, T[\times \mathbb{R})$, $\forall T \in \mathbb{R}_{+}$also exists, for the water-bag model corresponding to the initial conditions $\left(p_{0, \varepsilon}^{+}, p_{0, \varepsilon}^{-}, E_{0, \varepsilon}\right)$. Proposition 1.1 implies that $\mathbf{1}_{\left\{p_{\varepsilon}^{-}(t, x)<p<p_{\varepsilon}^{+}(t, x)\right\}}$ weakly solves the Vlasov equation associated to the electric field $\tilde{E}_{\varepsilon}$. Combining this with the uniqueness of the weak solution for the Vlasov-Maxwell system yields the equalities

$$
f_{\varepsilon}(t, x, p)=\mathbf{1}_{\left\{p_{\varepsilon}^{-}(t, x)<p<p_{\varepsilon}^{+}(t, x)\right\}}, E_{\varepsilon}(t, x)=\tilde{E}_{\varepsilon}(t, x) .
$$

We claim that $\left(p_{\varepsilon}^{-}\right)_{\varepsilon>0},\left(p_{\varepsilon}^{+}\right)_{\varepsilon>0}$ are relatively compact in $L_{\text {loc }}^{1}(] 0, T[\times \mathbb{R})$ and $\left(f_{\varepsilon}\right)_{\varepsilon>0}$ is relatively compact in $L_{\text {loc }}^{1}(] 0, T\left[\times \mathbb{R}_{x} ; L^{1}\left(\mathbb{R}_{p}\right)\right)$. As $p_{\varepsilon}^{-}, p_{\varepsilon}^{+}$are smooth, the considerations for entropy solutions apply and thus we deduce the uniform estimates with respect to $\varepsilon>0$

$$
\begin{aligned}
&\left\|E_{\varepsilon}(t)\right\|_{L^{\infty}} \leq\left(\left\|E_{0, \varepsilon}\right\|_{L^{\infty}}+T\left(\left\|p_{0, \varepsilon}^{+}\right\|_{L^{\infty}}+\left\|p_{0, \varepsilon}^{-}\right\|_{L^{\infty}}\right)\right) e^{2 T t} \\
& \leq\left(\left\|E_{0}\right\|_{L^{\infty}}+T\left(\left\|p_{0}^{+}\right\|_{L^{\infty}}+\left\|p_{0}^{-}\right\|_{L^{\infty}}\right)\right) e^{2 T t}=e_{T}(t) \\
&\left\|p_{\varepsilon}^{ \pm}(t)\right\|_{L^{\infty}} \leq\left\|p_{0, \varepsilon}^{ \pm}\right\|_{L^{\infty}}+\int_{0}^{t}\left\|E_{\varepsilon}(s)\right\|_{L^{\infty}} d s \leq\left\|p_{0}^{ \pm}\right\|_{L^{\infty}}+\int_{0}^{t} e_{T}(s) d s
\end{aligned}
$$




$$
\begin{aligned}
\max \left\{\left\|\partial_{t} E_{\varepsilon}(t)\right\|_{L^{\infty}},\left\|\partial_{x} E_{\varepsilon}(t)\right\|_{L^{\infty}}\right\} & \leq\left\|p_{\varepsilon}^{-}(t)\right\|_{L^{\infty}}+\left\|p_{\varepsilon}^{+}(t)\right\|_{L^{\infty}} \\
& \leq\left\|p_{0}^{-}\right\|_{L^{\infty}}+\left\|p_{0}^{+}\right\|_{L^{\infty}}+2 \int_{0}^{T} e_{T}(s) d s
\end{aligned}
$$

By the contraction property (2.4) we have for any $h, R>0$

$$
\begin{aligned}
& \int_{\mathbb{R}}\left|p_{\varepsilon}^{ \pm}(t, x+h)-p_{\varepsilon}^{ \pm}(t, x)\right| \mathbf{1}_{\{|x|<R\}} d x \leq \\
& \quad \int_{\mathbb{R}}\left|p_{0, \varepsilon}^{ \pm}(x+h)-p_{0, \varepsilon}^{ \pm}(x)\right| \mathbf{1}_{\{|x|<R+t\}} d x \\
& +\int_{0}^{t} \int_{\mathbb{R}}\left|E_{\varepsilon}(s, x+h)-E_{\varepsilon}(s, x)\right| \mathbf{1}_{\{|x|<R+t-s\}} d x d s .
\end{aligned}
$$

Since $p_{0, \varepsilon}^{ \pm}$are nondecreasing and bounded, they have bounded variation

$$
T V\left(p_{0, \varepsilon}^{ \pm}\right) \leq 2\left\|p_{0, \varepsilon}^{ \pm}\right\|_{L^{\infty}} \leq 2\left\|p_{0}^{ \pm}\right\|_{L^{\infty}} .
$$

Similarly, since $E_{\varepsilon}(t, \cdot)$ is nondecreasing and bounded we deduce that

$$
T V\left(E_{\varepsilon}(t, \cdot)\right) \leq 2\left\|E_{\varepsilon}(t)\right\|_{L^{\infty}} \leq 2 e_{T}(t), t \in[0, T], \varepsilon>0
$$

and therefore by (2.5), (2.6) one obtains

$$
\sup _{\varepsilon>0} T V\left(p_{\varepsilon}^{ \pm}(t)\right) \leq \sup _{\varepsilon>0}\left\{T V\left(p_{0, \varepsilon}^{ \pm}\right)+\int_{0}^{t} T V\left(E_{\varepsilon}(s)\right) d s\right\}<+\infty
$$

and

$$
\begin{aligned}
\int_{\mathbb{R}}\left|p_{\varepsilon}^{ \pm}(h, x)-p_{0, \varepsilon}^{ \pm}(x)\right| \mathbf{1}_{\{|x|<R\}} d x \leq & h\left\{T V\left(p_{0, \varepsilon}^{ \pm}\right)+\int_{0}^{h} T V\left(E_{\varepsilon}(s)\right) d s\right\} \\
& +2 R \int_{0}^{h}\left\|E_{\varepsilon}(s)\right\|_{L^{\infty}} d s .
\end{aligned}
$$

Applying the contraction property (2.4) once more, we obtain

$$
\begin{aligned}
\int_{\mathbb{R}}\left|p_{\varepsilon}^{ \pm}(t+h, x)-p_{\varepsilon}^{ \pm}(t, x)\right| \mathbf{1}_{\{|x|<R\}} d x \leq & \int_{\mathbb{R}}\left|p_{\varepsilon}^{ \pm}(h, x)-p_{0, \varepsilon}^{ \pm}(x)\right| \mathbf{1}_{\{|x|<R+t\}} d x \\
& +\int_{0}^{t} \int_{\mathbb{R}}\left|E_{\varepsilon}(s+h, x)-E_{\varepsilon}(s, x)\right| \mathbf{1}_{\{|x|<R+t-s\}} d x d s .
\end{aligned}
$$

Combining (3.5), (3.6), (3.7) and taking into account that $\sup _{\varepsilon>0}\left\|E_{\varepsilon}\right\|_{W^{1, \infty}(] 0, T[\times \mathbb{R})}<$ $+\infty$, it is easily seen that $\left(p_{\varepsilon}^{ \pm}\right)_{\varepsilon>0}$ are relatively compact in $L_{\text {loc }}^{1}(] 0, T[\times \mathbb{R})$. Therefore, up to a sequence extraction, we have $\lim _{\varepsilon} \backslash 0$ 传 $=p^{ \pm}$in $L_{\text {loc }}^{1}(] 0, T[\times \mathbb{R})$ for some bounded nondecreasing functions $p^{ \pm}$. We denote the density $f(t, x, p)=$ $\mathbf{1}_{\left\{p^{-}(t, x)<p<p^{+}(t, x)\right\}}$ by $f$. Obviously we have

$$
\begin{aligned}
\int_{\mathbb{R}}\left|f_{\varepsilon}-f\right| d p & =\int_{\mathbb{R}}\left|\mathbf{1}_{\left\{p_{\varepsilon}^{-}<p\right\}} \mathbf{1}_{\left\{p<p_{\varepsilon}^{+}\right\}}-\mathbf{1}_{\left\{p^{-}<p\right\}} \mathbf{1}_{\left\{p<p^{+}\right\}}\right| d p \\
& \leq \int_{\mathbb{R}}\left\{\left|\mathbf{1}_{\left\{p_{\varepsilon}^{-}<p\right\}}-\mathbf{1}_{\left\{p^{-}<p\right\}}\right|+\left|\mathbf{1}_{\left\{p<p_{\varepsilon}^{+}\right\}}-\mathbf{1}_{\left\{p<p^{+}\right\}}\right|\right\} d p \\
& =\left|p_{\varepsilon}^{-}(t, x)-p^{-}(t, x)\right|+\left|p_{\varepsilon}^{+}(t, x)-p^{+}(t, x)\right|,
\end{aligned}
$$


implying that $\lim _{\varepsilon \searrow 0} f_{\varepsilon}=f$ in $L_{\text {loc }}^{1}(] 0, T\left[\times \mathbb{R}_{x} ; L^{1}\left(\mathbb{R}_{p}\right)\right.$ ) (up to the same sequence extraction). Moreover, eventually performing a new extraction, we deduce by the ArzelaAscoli theorem that $\left(E_{\varepsilon}\right)_{\varepsilon>0}$ converges uniformly on compact sets towards some electric field $E \in W^{1, \infty}(] 0, T[\times \mathbb{R})$. Combining all these convergences it is easily seen that the limit functions $\left.\left(p^{ \pm}, f=\mathbf{1}_{\left\{p^{-}<p<p^{+}\right\}}\right), E\right)$ weakly solve both the water-bag model and the Vlasov-Maxwell system. Therefore we have proved that the unique weak solution of the Vlasov-Maxwell system is $\left(f=\mathbf{1}_{\left\{p^{-}<p<p^{+}\right\}}, E\right)$, where $\left(p^{ \pm}, E\right)$ is a weak solution for the water-bag model.

Acknowledgement. JAC is partially supported by the project MTM200806349-C03-03 from DGI-MICINN (Spain) and 2005SGR00611.

\section{REFERENCES}

[1] A. Arseneev, Global existence of a weak solution of the Vlasov system of equations, U.R.S.S. Comp. Math. Phys., 15, 131-143, 1975.

[2] P. Bertrand and M.R. Feix, Nonlinear electron plasma oscillations: the "water bag model", Phys. Lett. A, 28, 68-69, 1968.

[3] M. Bostan, Asymptotic behavior of weak solutions for the relativistic Vlasov-Maxwell equations with large light speed, J. Differ. Eqs., 227, 444-498, 2006.

[4] M. Bostan, Mild solutions for the relativistic Vlasov-Maxwell system for laser-plasma interaction, Quart. Appl. Math., 65, 163-187, 2007.

[5] F. Bouchut, F. Golse and C. Pallard, Classical solutions and the Glassey-Strauss theorem for the 3D Vlasov-Maxwell system, Arch. Ration. Mech. Anal., 170, 1-15, 2003.

[6] N. Besse, P. Bertrand, F. Berthelin, Y. Brenier, P. Morel and E. Gravier, The multi-water-bag model for collisionless kinetic equations, preprint.

[7] Y. Brenier and L. Corrias, A kinetic formulation for multi-branch entropy solutions of scalar conservation laws, Ann. Inst. H. Poincaré Anal. Non Linéaire, 15, 169-190, 1998.

[8] J.A. Carrillo and S. Labrunie, Global solutions for the one-dimensional Vlasov-Maxwell system for laser-plasma interaction, Math. Models Meth. Appl. Sci., 16, 19-57, 2006.

[9] P. Degond, Local existence of solutions of the Vlasov-Maxwell equations and convergence to the Vlasov-Poisson equations for infinite light velocity, Math. Meth. Appl. Sci., 8, 533-558, 1986.

[10] R.J. DiPerna and P.L. Lions, Global weak solutions of the Vlasov-Maxwell system, Comm. Pure Appl. Math., XVII, 729-757, 1989.

[11] L.C. Evans, Partial Differential Equations, Graduate Studies in Mathematics, American Mathematical Society, Providence Rhode Island, 19, 2002.

[12] R. Glassey and W. Strauss, Singularity formation in a collisionless plasma could only occur at high velocities, Arch. Ration. Mech. Anal., 92, 56-90, 1986.

[13] E. Godlewski and P.A. Raviart, Hyperbolic systems of conservation laws, Mathématiques et Applications, Ellipses, 1991.

[14] L. Gosse, Using K-branch entropy solutions for multivalued geometric optics computations, J. Comput. Phys., 180, 155-182, 2002.

[15] L. Gosse and O. Runborg, Finite moment problems and applications to multiphase computations in geometric optics, Commun. Math. Sci., 3, 373-392, 2005.

[16] S. Klainerman and G. Staffilani, A new approach to study the Vlasov-Maxwell system, Commun. Pure Appl. Anal., 1, 103-125, 2002.

[17] P.L. Lions and B. Perthame, Propagation of moments and regularity for the 3-dimensional Vlasov-Poisson system, Invent. Math., 105, 415-430, 1991.

[18] P.L. Lions, B. Perthame and E. Tadmor, A kinetic formulation of multidimensional scalar conservation laws and related equations, J. Amer. Math. Soc., 7, 169-191, 1994.

[19] B. Perthame, Kinetic Formulation of Conservation Laws, Oxford University Press, 2002.

[20] K. Pfaffelmoser, Global classical solutions of the Vlasov-Poisson system in three dimensions for general initial data, J. Differ. Eqs., 95, 281-303, 1992. 